\title{
Dinâmica do Chumbo no Lago do Parque Ingá, Maringá, PR, Brasil
}

\author{
Sérgio Renato Vaz ${ }^{1}$, Ervim Lenzi ${ }^{1 *}$, Eduardo Bernardi Luchese ${ }^{2}$ e Luzia O. B. Fávero ${ }^{2}$ \\ 1 - Professor do Departamento de Química da Universidade Federal do Paraná e da Pontifícia Universidade \\ Católica do Paraná - Curitiba - Paraná ${ }^{2}$ - Professores do Departamento de Química, Universidade Estadual de \\ Maringá, Avenida Colombo, 5790 - Maringá - Paraná.
}

\begin{abstract}
This work introduce the study of lead dynamic in the lake of Ingá Park, Maringá - PR. Monthly, during a year, samples of water and sediments were collected and $\mathrm{pH}$ and temperature measured. In the water samples were determined the chemical oxygen demand (COD) and the total lead concentration in water and sediment samples. The determination of lead concentration in water and sediments was performed with atomic absorption spectrometer - flame technique. The monthly mean values found, presented in intervals and global mean values, were respectively: lead concentration in water $\left(n g . m L^{-1}\right)$, non detected $(n d)-55.56$ and 19.23; lead in sediments $\left(\mu g . g^{-1}\right), 48.73$ - 92.87 and 71.93; $p H, 7.02$ - 8.23 and 7.60; temperature $\left({ }^{\circ} \mathrm{C}\right), 19.33$ - 27.48 and $23.73 ; C O D$ (mg. $\left.L^{-1}\right), 13.33$ - 30.11 and 23.54. A survey of the lead concentration results found permit to conclude, at $5 \%$ of significance, that the lead concentration in water was independent of the sampling point (local), but dependent of the sampling period (temporality) and in the sediment dependent of the two parameters.
\end{abstract}

Key words: Lead, lakes, environmental pollution, elements environmental dynamic, environment

\section{INTRODUÇÃO}

O chumbo é um metal pesado e tem efeito tóxico na biota (Moore \& Ramamoorthy, 1984). O American Council of Governmental Industrial Hygienists (ACGIH), coloca seus compostos em grau máximo de toxidez (Sax, 1979 e Lewis, 1996). Nas pessoas causa a doença denominada saturnismo. A vida média do chumbo no sangue está entre 1 a 2 meses, nos ossos de 20 a 30 anos (Klaassen, 1991). Entre 1972 e 1979 2,7 milhões de crianças foram examinadas e 189.000 (ou 7\%) apresentaram níveis críticos de chumbo (Boekx, 1986).

$\mathrm{O}$ chumbo encontra-se naturalmente na crosta terrestre num valor médio de $12,5 \mathrm{mg} \cdot \mathrm{kg}^{-1}$ (Taylor, 1965) e 16 mg. $\mathrm{kg}^{-1}$ (Craig, 1980 e Lee, 1980). Contudo, é um dos metais que acompanha a civilização. Em 1981 era o quinto elemento no ranking mundial da produção de metais, perdendo apenas para o $\mathrm{Fe}, \mathrm{Cu}, \mathrm{Al}$ e $\mathrm{Zn}$ (Kirk-Othmer, 1981). A ação antrópica o espalhou no meio ambiente (Davies, 1990;
Purves, 1985; Daines et al., 1970; Schuck \& Locke, 1970 \& Smith et al. 1970). Hoje é um elemento ubíquo no ambiente, segundo Craig, 1980. Nos grandes rios americanos encontra-se no intervalo de concentração de 0 - $55 \mu \mathrm{g} . \mathrm{L}^{-1}$, com média 4,0 $\mu \mathrm{g} . \mathrm{L}^{-1}$ (Durum \& Haffty, 1963). Em águas não contaminadas é encontrado próximo ao valor de 3,0 $\mu \mathrm{g} . \mathrm{L}^{-1}$ (Moore \& Ramamoorthy, 1984). Nos solos varia de 10 a 100 mg.kg ${ }^{-1}$ (Davies, 1980 e Adriano, 1986). Nas plantas com folhas maduras, em peso seco, a concentração de $\mathrm{Pb}$ varia de 5,0 a $10 \mathrm{mg} \cdot \mathrm{kg}^{-1}$. Em níveis tóxicos para as plantas, encontra-se no intervalo de 30 a 300 mg.kg ${ }^{-1}$ (KabataPendias \& Pendias, 1992). Em animais encontra-se no intervalo de 0,0 a $0.35 \mathrm{mg} \cdot \mathrm{kg}^{-1}$. $\mathrm{Na}$ água do mar está na ordem de $3,0.10^{-5} \mathrm{mg} \cdot \mathrm{L}^{-1}$ (McGraw-Hill Esncyclopedia, 1980). Em sedimentos pelágicos encontra-se na ordem de 109 mg.kg ${ }^{-1}$ (Horne, 1969 e Chow \& Patterson, 1962). Além do mais é considerado um dos principais metais pesados poluentes da atmosfera (Rodricks, 1995). Em corpos de água usados para a irrigação, sedentação animal e

\footnotetext{
* Autor para correspondência
} 
aquicultura são recomendados os limites de 30 a $50 \mu \mathrm{g} . \mathrm{L}^{-1}$, Resolução $\quad \mathrm{N}^{\circ}$ 20/CONAMA (Conselho Nacional do Meio Ambiente).

Considerando que o lago (represa) do Parque do Ingá (Maringá - PR) está dentro da cidade de Maringá e é utilizado para lazer por milhares de pessoas que o visitam por ano, achou-se conveniente fazer nele um estudo do comportamento do metal pesado chumbo.

\section{MATERIAIS E MÉTODOS}

Locais e número de coletas de amostras de água (Bartram \& Ballance, 1996): Foram definidos 6 locais de coleta de amostras, conforme Figura 01. Os locais 1 e 2 correspondem a pontos rasos, perto dos quais o lago é alimentado por duas fontes. Os locais: $3 \mathrm{e}$ 4,5 e 6 , e 7 e 8 correspondem respectivamente à superfície e fundo do ponto. $\mathrm{O}$ ponto 9 corresponde ao local de escoamento do lago. Ao todo têm-se 6 amostras de superfície e 3 de fundo (50 $\mathrm{cm}$ do fundo). As amostras de fundo foram coletadas com o auxílio de uma garrafa de Van Dorn (Souza \& Derísio, 1977). Foram obedecidas as normas e cuidados preconizados pela literatura pertinente, em seus aspectos gerais (Keith, 1996a e WMO, 1988) e para: a limpeza do material (Keith, 1996b; Moody \& Lindstrom, 1977 e Karin et al., 1975), à conservação das amostras (Keith, 1996b; Mart, 1979 e Wagner, 1976) e para evitar a contaminação. (Patterson \& Settle, 1976).

Para a determinação do DQO foi coletado um litro de amostra composta, em cada ponto de coleta, estocado em frasco de polietileno e adicionado $\mathrm{H}_{2} \mathrm{SO}_{4}$ concentrado até um $\mathrm{pH}<2$. Após levado à geladeira a $4^{\circ} \mathrm{C}$ para a posterior análise.

Para a determinação do chumbo e outros metais pesados foi estocado um litro de amostra composta, em frasco de polietileno e conservado com $\mathrm{HNO}_{3}$ concentrado até $\mathrm{pH}<2$. No ato da coleta foram medidos o $\mathrm{pH}$ e a temperatura das amostras.
Locais de coleta das amostras de sedimento (Keith, 1996a): Foram definidos 6 pontos para a coleta de sedimentos correspondendo aos 6 locais de coleta de amostras de água de superfície, conforme Figura 01. Na coleta dos sedimentos utilizou-se uma draga de Van Veen. (Souza \& Derísio, 1977). Uma alíquota de aproximadamente $2 \mathrm{~kg}$ de sedimentos de cada local de 0 a $10 \mathrm{~cm}$ da parte superior do fundo (Polprasert, 1982) foi coletada e estocada em sacos plásticos e levada ao freezer $\left(-10^{\circ} \mathrm{C}\right)$ para posteriores análises (Agemian \& Chau, 1975).

Análise da água: Determinação do Chumbo As amostras foram inicialmente preconcentradas com a redução do volume 5 vezes em banhomaria e ao mesmo tempo digeridas, por via úmida, com alíquotas de $\mathrm{HNO}_{3}$ concentrado. Após, a concentração de chumbo foi determinada pelo método da absorção atômica modalidade chama (Horwitz, 1980). O espectrômetro de absorção atômica utilizado foi o da CG Instrumentos Científicos Ltda., Modelo CG AA 7000 ABC. O branco e os padrões utilizados na curva analítica foram submetidos ao mesmo tratamento das amostras. O limite de detecção do método foi $c_{\mathrm{L}}=11 \mu \mathrm{g} \cdot \mathrm{L}^{-1}$ (Analytical Methods Committee, 1987 e Welz, 1985).

Demanda Química de Oxigênio (DQO) - A matéria orgânica das amostras foi oxidada com dicromato de potássio, em excesso, em ácido sulfúrico concentrado e sulfato de prata (catalisador), em ebulição em balão com refluxo. Após resfriamento foi titulado o excesso de dicromato de potássio. O branco foi submetido ao mesmo processo. A seguir foram feitos os cálculos (Braile \& Cavalcanti, 1979).

Análise dos sedimentos: Preparação das amostras - As amostras foram descongeladas e transferidas para bacias rasas de plástico e colocadas para secar em ambiente aberto, arejado e protegido de contaminação. Após foram moídas em geral de porcelana adequado, peneiradas em tamis de 0,5 $\mathrm{mm}$ de malha, submetidas a 
Tabela 01. Quadro da análise de variância dos valores das concentrações de chumbo total nas amostras de água coletadas nos 12 meses.

\begin{tabular}{|c|c|c|c|c|c|}
\hline fonte de variação & $\begin{array}{l}\text { Graus de } \\
\text { liberdade }\end{array}$ & $\begin{array}{l}\text { Soma dos } \\
\text { quadrados }\end{array}$ & $\begin{array}{l}\text { Quadrados } \\
\text { médios }\end{array}$ & $\begin{array}{c}\text { Valor de } \\
\text { F }\end{array}$ & Prob $>F$ \\
\hline Local & 8 & 2464,060 & 308,007 & 0,982 & 0,544 \\
\hline Meses & 11 & 38431,717 & 3493,792 & 11,141 & 0,00001 \\
\hline Resíduo & 88 & 27597,220 & 313,605 & & \\
\hline Total & 107 & 68492,997 & & & \\
\hline
\end{tabular}

Média global = 19,23 ng.mL ${ }^{-1} ;$ Coeficiente de variação $=92,10 \%$.

quarteação, transferidas para cápsulas de porcelana de 100-ml de capacidade e colocadas em estufa por 6 horas a $110{ }^{\circ} \mathrm{C}$. A seguir retiradas, esfriadas em dissecador e estocadas em frascos de polietileno para posterior análises.

Digestão - Transferiu-se 0,5000 g de amostra (ou valor exato próximo deste) para um tubo de ensaio com haste longa $(2,5 \mathrm{~cm}$ de diâmetro $\mathrm{x}$ $25 \mathrm{~cm}$ de comprimento), com uma tapa de vidro e adicionaram-se 5 gotas de $\mathrm{H}_{2} \mathrm{O}_{2}$ a $30 \%$ e $2 \mathrm{~mL}$ de $\mathrm{HNO}_{3}$ concentrado. $\mathrm{O}$ sistema foi levado ao bloco digestor com um aumento gradativo de temperatura a $170{ }^{\circ} \mathrm{C}$ até que o volume se reduzisse a metade. Resfriou-se e adicionou-se $1 \mathrm{ml}$ de $\mathrm{HClO}_{4}$ a $60 \%$. O sistema retornou ao aquecimento até a temperatura de $170^{\circ} \mathrm{C}$, ali permanecendo até desaparecer os vapores esbranquiçados. Após retirou-se o tubo do bloco digestor, resfriou-se e com o auxílio de um funil transferiu-se quantitativamente o conteúdo do tubo de ensaio para um balão volumétrico de 50 $\mathrm{ml}$ e completou-se o volume com água destilada e deionizada (Sinex et al.,1980 e Krishnamurty et al., 1976). As concentrações de chumbo foram lidas com o auxílio do espectrômetro de absorção atômica, na modalidade chama. As soluções branco e padrões da curva analítica foram submetidas ao mesmo processo que as amostras.

Análise estatística: Os dados experimentais foram submetidos a uma análise estatística de variância e de correlação linear (Gomes, 1987 e Costa Neto, 1977).

\section{RESULTADOS E DISCUSSÃO}

Os valores das concentrações de chumbo total na água, em ng.mL ${ }^{-1}$, compõem uma matriz de 9 linhas (locais de coleta no lago) e 12 colunas (meses de coleta - temporalidade). A Tabela 01 apresenta o quadro de análise de variância dos dados.

Pela análise verifica-se que entre os pontos de coleta em nível de $5 \%$ e $1 \%$ de significância, não há diferença significativa. Ao passo que entre os meses, que expressam a temporalidade das coletas, há diferença significativa.

Tabela 02. Quadro da análise de variância das concentrações de chumbo nos sedimentos coletados nos 12 meses.

\begin{tabular}{lccccr}
\hline fonte de variação & $\begin{array}{c}\text { Graus de } \\
\text { liberdade }\end{array}$ & $\begin{array}{c}\text { Soma dos } \\
\text { quadrados }\end{array}$ & $\begin{array}{c}\text { Quadrados } \\
\text { médios }\end{array}$ & $\begin{array}{c}\text { Valor de } \\
\text { F }\end{array}$ & Prob $>$ F \\
\cline { 1 - 3 } Local & 5 & 3233,471 & 646,694 & 4,052 & 0,00364 \\
Meses & 11 & 13593,005 & 1235,728 & 7,743 & 0,0001 \\
Resíduo & 55 & 8777,588 & 159,592 & & \\
\cline { 1 - 3 } Total & 71 & 25604,064 & & & \\
\hline
\end{tabular}

Média global $=71,93 \mu \cdot \mathrm{g}^{-1} ;$ Coeficiente de variação $=17,56 \%$. 
Os resultados da concentração de chumbo nos sedimentos, em $\mu \mathrm{g} . \mathrm{g}^{-1}$, compõem uma matriz com 6 linhas (locais de coleta) e 12 colunas (meses em que se realizaram as coletas). A Tabela 02 mostra o quadro de análise de variância dos resultados. Nela pode-se verificar que tanto a $5 \%$ quanto a $1 \%$ de significância há diferença significativa nos valores médios da concentração de chumbo encontrados nos locais (pontos) e nos meses de coletas.

Análise da influência da temporalidade: A Tabela 03 apresenta os valores médios mensais das concentrações de chumbo na água e nos sedimentos, dos 9 locais amostrados, ao longo de 12 meses. Mostra também os valores de: I.P. (Índice Pluviométrico), $\mathrm{pH}$, temperatura, alcalinidade e DQO (Demanda Química de Oxigênio). A Tabela 04 apresenta o estudo da correlação destas variáveis com a concentração de $\mathrm{Pb}$ na água $-\mathrm{C}_{\mathrm{Pb}(\mathrm{a})}$ e no sedimento $-\mathrm{C}_{\mathrm{Pb}(\mathrm{s})}$.

Pela Tabela 04 verifica-se que existe uma correlação positiva da $\mathrm{C}_{\mathrm{Pb}(\mathrm{a})}$ com Índice Pluviométrico (I.P.) em nível de 5\% de significância, o que era esperado pelos seguintes motivos: - o lago está dentro da cidade e cercado por avenidas, ver Figura 01, onde trafegam milhares de veículos por mês, deixando no asfalto e vizinhanças os particulados provenientes da queima do combustível em que se encontram também resíduos de chumbo. A água da enxurrada lava o asfalto e vizinhanças, dissolvendo compostos de $\mathrm{Pb}$ e carreando particulados para o lago.

Ta bela 03. Valores médios mensais da concentração total de $\mathrm{Pb}$ na água - $\left(\mathrm{C}_{\mathrm{Pb}(\mathrm{a})}\right)$, nos sedimentos $\left(\mathrm{C}_{\mathrm{Pb}(\mathrm{s})}\right), \mathrm{pH}, \mathrm{t}, \mathrm{Alc}$., DQO e I.P.

\begin{tabular}{|c|c|c|c|c|c|c|c|c|c|}
\hline \multirow{2}{*}{ Mês } & \multicolumn{2}{|c|}{$\mathrm{C}_{\mathrm{Pb}(\mathrm{a})} \quad\left(\mathrm{ng} \cdot \mathrm{mL}^{-1}\right)$} & $\mathrm{C}_{\mathrm{Pb}(\mathrm{s})}$ & \multirow[t]{2}{*}{$\begin{array}{l}\left(\mu \mathrm{g} \cdot \mathrm{g}^{-1}\right) \\
-\quad \mathrm{mm}(\mathrm{mês})^{-1}\end{array}$} & \multirow[t]{2}{*}{ I.P. } & \multirow[t]{2}{*}{$\underset{{ }^{\circ} \mathrm{CH}}{\mathrm{pH}}$} & \multirow{2}{*}{$\begin{array}{r}\mathrm{T} \\
\mathrm{mg.L}^{-1} \\
\mathrm{CaCO}_{3}\end{array}$} & \multirow{2}{*}{$\begin{array}{l}\text { Alc } \\
\text { mg. } \mathrm{L}^{-1} \\
{\text { de } \mathrm{O}_{2}}\end{array}$} & \multirow[t]{2}{*}{ DQO } \\
\hline & $\mathrm{m}$ & $\mathrm{s}$ & $\mathrm{m}$ & & & & & & \\
\hline Mar. & 40,31 & 31,26 & 81,98 & 6,48 & 121,3 & 7,51 & 26,50 & 39,93 & 14,64 \\
\hline Abr. & 28,33 & 30,95 & 78,58 & 3,19 & 105,3 & 7,77 & 22,92 & 41,19 & 30,11 \\
\hline Mai. & 5,22 & 8,10 & 76,93 & 17,76 & 28,3 & 7,56 & 21,51 & 39,36 & 13,33 \\
\hline Jun. & 0,00 & 0,00 & 85,25 & 21,76 & 84,1 & 7,51 & 19,33 & 36,15 & 27,09 \\
\hline Jul. & 0,00 & 0,00 & 92,87 & 10,21 & 71,7 & 8,23 & 20,33 & 36,51 & 23,62 \\
\hline Ago. & 10,76 & 9,49 & 71,03 & 16,61 & 13,0 & 8,22 & 22,50 & 38,33 & 29,53 \\
\hline Set. & 49,00 & 24,74 & 85,67 & 9,74 & 197,2 & 7,33 & 20,33 & 42,45 & 26,89 \\
\hline Out. & 55,56 & 20,29 & 52,90 & 9,55 & 248,9 & 7,37 & 22,96 & 36,24 & 26,51 \\
\hline Nov. & 8,44 & 8,47 & 71,37 & 20,47 & 94,2 & 7,80 & 27,48 & 44,73 & 27,36 \\
\hline Dez. & 22,67 & 13,44 & 64,07 & 15,88 & 172,3 & 7,55 & 26,94 & 45,53 & 21,57 \\
\hline Jan. & 7,33 & 19,18 & 53,83 & 14,95 & 242,6 & 7,02 & 26,61 & 41,28 & 20,81 \\
\hline Fev. & 3,11 & 4,81 & 48,73 & 10,02 & 140,7 & 7,30 & 27,37 & 38,33 & 21,03 \\
\hline $\mathrm{mg}$ & 19,23 & & 71,93 & & 126,6 & 7,60 & 23,73 & 40,00 & 23,54 \\
\hline
\end{tabular}

m - média aritmética; s - desvio padrão; I.P. - Índice Pluviométrico; $\mathrm{t}$ - temperatura; Alc - alcalinidade (Vaz, 1996); DQO - Demanda Química de Oxigênio; mg - média global; $\mathrm{C}_{\mathrm{Pb}(\mathrm{s})}$ - Concentração de chumbo nos sedimentos; $\mathrm{C}_{\mathrm{Pb}(\mathrm{a})}$ - Concentração de chumbo total na água. 
Tabela 04. Quadro da análise da correlação linear, em nível de 5\% de significância, entre as concentrações de $\mathrm{Pb}$ na água - $\mathrm{C}_{\mathrm{Pb}(\mathrm{a})}$ - e as concentrações de $\mathrm{Pb}$ nos sedimentos com outras variáveis do meio, em função da época da coleta.

\begin{tabular}{|c|c|c|c|c|c|}
\hline Variável & & Equação da correlação & $\mathrm{R}$ & $\mathrm{S}_{\mathrm{r}}$ & Correlação \\
\hline \multicolumn{6}{|c|}{1 - Correlação das variáveis com $\mathrm{C}_{\mathrm{Pb}(\mathrm{a})}$} \\
\hline I.P. & $\mathrm{C}_{\mathrm{Pb}(\mathrm{a})}$ & $=0.899+0,145 .($ I.P. $)$ & 0,563 & 0,261 & $\overline{\text { positiva }}$ \\
\hline $\mathrm{pH}$ & $\mathrm{C}_{\mathrm{Pb}(\mathrm{a})}$ & $=139-15,7 .(\mathrm{pH})$ & $-0,288$ & 0,303 & sem relação \\
\hline $\mathrm{T}$ & $\mathrm{C}_{\mathrm{Pb}(\mathrm{a})}$ & $=21,3-0,0870 .(\mathrm{t})$ & $-0,0136$ & 0,316 & sem relação \\
\hline Alc & $\mathrm{C}_{\mathrm{Pb}(\mathrm{a})}$ & $=-13,6+0,821 .(\mathrm{Alc})$ & 0,131 & 0,313 & sem relação \\
\hline DQO & $\mathrm{C}_{\mathrm{Pb}(\mathrm{a})}$ & $=10,1+0,389 .(\mathrm{DQO})$ & 0,108 & 0,314 & sem relação \\
\hline \multicolumn{6}{|c|}{2 - Correlação das variáveis com $\mathrm{C}_{\mathrm{Pb}(\mathrm{s})}$} \\
\hline I.P. & $\mathrm{C}_{\mathrm{Pb}(\mathrm{s})}$ & $=84,5-0,0995 .($ I.P.) & $-0,531$ & 0,268 & $\overline{\text { negativa }}$ \\
\hline $\mathrm{pH}$ & $\mathrm{C}_{\mathrm{Pb}(\mathrm{s})}$ & $=-87,6+21,0 .(\mathrm{pH})$ & 0,526 & 0,269 & positiva \\
\hline $\mathrm{T}$ & $\mathrm{C}_{\mathrm{Pb}(\mathrm{s})}$ & $=144-3,02 .(\mathrm{t})$ & 0,646 & 0,241 & positiva \\
\hline Alc & $\mathrm{C}_{\mathrm{Pb}(\mathrm{s})}$ & $=88,1-0,405 .(\mathrm{Alc})$ & $-0,088$ & 0,315 & sem relação \\
\hline DQO & $\mathrm{C}_{\mathrm{Pb}(\mathrm{s})}$ & $=67,8+0,175 .(\mathrm{DQO})$ & 0,0665 & 0,316 & sem relação \\
\hline $\mathrm{C}_{\mathrm{Pb}(\mathrm{a})}$ & $\mathrm{C}_{\mathrm{Pb}(\mathrm{s})}$ & $=72,7-0,0381\left(\mathrm{C}_{\mathrm{Pb}(\mathrm{a})}\right)$ & $-0,0523$ & 0,316 & sem relação \\
\hline
\end{tabular}

$\mathrm{r}$ - coeficiente de correlação linear; $\mathrm{s}_{\mathrm{r}}$ - desvio padrão de r; I.P. - Índice Pluviométrico; $\mathrm{t}$ temperatura; Alc - Alcalinidade DQO - Demanda Química de Oxigênio.

Tabela 05. Valores médios das concentrações de $\mathrm{Pb}$ nas amostras de água $-\mathrm{C}_{\mathrm{Pb}(\mathrm{ag})}$, valores médios das concentrações de $\mathrm{Pb}$ nos sedimentos - $\mathrm{C}_{\mathrm{Pb}(\mathrm{sd})}$, e os valores médios das medidas de $\mathrm{pH}$, temperatura, Alcalinidade e DQO, de cada local de amostragem.

\begin{tabular}{|c|c|c|c|c|c|c|c|c|}
\hline \multirow{2}{*}{$\begin{array}{l}\text { Lo- } \\
\text { Cais }\end{array}$} & \multicolumn{2}{|c|}{$\mathrm{C}_{\mathrm{Pb}(\mathrm{ag})}$ (ng. $\left.\mathrm{mL}^{-1}\right)$} & \multicolumn{2}{|c|}{$\mathrm{C}_{\mathrm{Pb}(\mathrm{sd})}\left(\mu \mathrm{g} \cdot \mathrm{g}^{-1}\right)$} & \multirow[t]{2}{*}{$\mathrm{pH}$} & \multirow{2}{*}{$\begin{array}{r}\mathrm{t} \\
{ }^{\circ} \mathrm{C}\end{array}$} & \multirow{2}{*}{$\begin{array}{c}\text { Alc } \\
\mathrm{mg.L}^{-1} \\
\mathrm{CaCO}_{3}\end{array}$} & \multirow{2}{*}{$\begin{array}{l}\text { DQO } \\
\text { mg.L }{ }^{-1} \\
\mathrm{O}_{2}\end{array}$} \\
\hline & $\mathrm{m}$ & $\mathrm{S}_{\mathrm{r}}$ & $\mathrm{m}$ & $\mathrm{S}_{\mathrm{r}}$ & & & & \\
\hline $1 *$ & 20,88 & 23,49 & 68,21 & 19,46 & 7,46 & 23,30 & 36,47 & 21,26 \\
\hline $2 *$ & 20,30 & 23,51 & 76,87 & 14,51 & 7,69 & 23,69 & 36,28 & 21,77 \\
\hline $3 *$ & 16,82 & 24,00 & $\ldots$ & $\ldots$ & 7,75 & 23,81 & 36,38 & 17,81 \\
\hline $4 * *$ & 22,87 & 29,71 & 72,51 & 19,14 & 7,35 & 23,43 & 43,59 & 26,88 \\
\hline $5^{*}$ & 13,45 & 23,69 & $\ldots$ & $\ldots$ & 7,86 & 24,21 & 34,85 & 23,47 \\
\hline $6 * *$ & 29,05 & 37,53 & 78,58 & 20,09 & 7,34 & 23,29 & 43,59 & 23,06 \\
\hline $7 *$ & 17,15 & 21,82 & $\ldots$ & $\ldots$ & 7,87 & 24,31 & 36,54 & 22,50 \\
\hline $8 * *$ & 20,18 & 26,01 & 76,43 & 18,00 & 7,15 & 22,78 & 56,29 & 34,12 \\
\hline $9 *$ & 12,35 & 17,79 & 59,03 & 18,72 & 7,91 & 24,78 & 36,04 & 21,01 \\
\hline $\mathrm{Mg}$ & 19,23 & & 71,93 & & 7,60 & 23,73 & 40,00 & 233,54 \\
\hline
\end{tabular}

* - amostra de superfície; ** - amostra de fundo (50 cm do fundo do lago); - $\mathrm{m}$ - média aritmética; $\mathrm{s}_{\mathrm{r}}$

- desvio padrão; t - temperatura; Alc - Alcalinidade (Vaz, 1996); DQO - Demanda Química de

Oxigênio; mg - média global; ... - amostra não existente. 
Tabela 06. Quadro da análise da correlação linear, em nível de 5\% de significância, entre os valores das concentrações de $\mathrm{Pb}$ na água - $\mathrm{C}_{\mathrm{Pb}(\mathrm{ag})}$, e dos valores das concentrações de $\mathrm{Pb}$ nos sedimentos $-\mathrm{C}_{\mathrm{Pb}(\mathrm{sd})}$, com as variáveis do meio: $\mathrm{pH}$, temperatura, alcalinidade e $\mathrm{DQO}$, em função dos locais de amostragem.

\begin{tabular}{|c|c|c|c|c|}
\hline Variável & Equação da correlação & $\mathrm{r}$ & $\mathrm{S}_{\mathrm{r}}$ & Correlação \\
\hline \multicolumn{5}{|c|}{1 - Correlação das variáveis do meio com $\mathrm{C}_{\mathrm{Pb}(\mathrm{ag})}$} \\
\hline $\mathrm{pH}$ & $\mathrm{C}_{\mathrm{Pb}(\mathrm{ag})}=124-13,86 .(\mathrm{pH})$ & $-0,761$ & 0,245 & negativa \\
\hline $\mathrm{t}$ & $\mathrm{C}_{\mathrm{Pb}(\mathrm{ag})}=163-6,06 .(\mathrm{t})$ & $-0,738$ & 0,255 & negativa \\
\hline Alc & $\mathrm{C}_{\mathrm{Pb}(\mathrm{ag})}=6,03+0,330 .(\mathrm{Alc})$ & 0,452 & 0,337 & sem relação \\
\hline DQO & $\mathrm{C}_{\mathrm{Pb}(\mathrm{ag})}=13,0+0,265 .(\mathrm{DQO})$ & 0,243 & 0,366 & sem relação \\
\hline \multicolumn{5}{|c|}{2 - Correlação das variáveis do meio com $\mathrm{C}_{\mathrm{Pb}(\mathrm{sd})}$} \\
\hline $\mathrm{pH}$ & $\mathrm{C}_{\mathrm{Pb}(\mathrm{sd})}=210-18,5 .(\mathrm{pH})$ & $-0,689$ & 0,274 & negativa \\
\hline $\mathrm{t}$ & $\mathrm{C}_{\mathrm{Pb}(\mathrm{sd})}=274-8,59 .(\mathrm{t})$ & $-0,789$ & 0,232 & negativa \\
\hline DQO & $\mathrm{C}_{\mathrm{Pb}(\mathrm{sd})}=57,0+0,604 .(\mathrm{DQO})$ & 0,420 & 0,343 & sem relação \\
\hline Alc & $\mathrm{C}_{\mathrm{Pb}(\mathrm{sd})}=55,3+0,114 .(\mathrm{Alc})$ & 0,0337 & 0,378 & sem relação \\
\hline $\mathrm{C}_{\mathrm{Pb}(\mathrm{ag})}$ & $\mathrm{C}_{\mathrm{Pb}(\mathrm{sd})}=48,8+1,11 .\left[\mathrm{C}_{\mathrm{Pb}(\mathrm{ag})}\right]$ & 0,809 & 0,294 & positiva \\
\hline
\end{tabular}

$\mathrm{r}$ - coeficiente de correlação linear; $\mathrm{s}_{\mathrm{r}}$ - desvio padrão de $\mathrm{r} ; \mathrm{pH}--\log \left[\mathrm{H}^{+}\right] ; \quad \mathrm{t}$ -

temperatura; Alc - Alcalinidade (Vaz, 1996); DQO - Demanda Química de Oxigênio.

Conforme Tabela 04 (1 - Correlação das variáveis do meio com $\mathrm{C}_{\mathrm{Pb}(\mathrm{a})}$ ) não se verificou correlação linear com as variáveis: $\mathrm{pH}$, temperatura, alcalinidade e DQO.

Entre a concentração de $\mathrm{Pb}$ nos sedimentos $\mathrm{C}_{\mathrm{Pb}(\mathrm{s})}$ e as variáveis estudadas, Tabela 04 (2Correlação das variáveis do meio com $\mathrm{C}_{\mathrm{Pb}(\mathrm{s})}$ ) verifica-se em nível de 5\% de significância:

- Há uma correlação negativa com I.P., que é explicada devido ao revolvimento da camada superior dos sedimentos, levando-a para o meio do corpo de água, quando há movimentação do corpo d'água;

- Há uma correlação positiva com o pH. O fato é devido ao equilíbrio $\mathrm{Pb}(\mathrm{OH})_{2(\mathrm{ppt})}=\mathrm{Pb}^{2+}+2$. $\mathrm{OH}$ que com um aumento do $\mathrm{pH}$, isto é, com o acréscimo de ${ }^{-} \mathrm{OH}$, desloca-se para a formação do precipitado $\mathrm{Pb}(\mathrm{OH})_{2(\mathrm{ppt})}$ e vice-versa.

- Há uma correlação positiva com a temperatura (t) provavelmente devido a estratificação térmica que ocorre, conforme pode ser verificado na Tabela 07, onde as amostras de fundo apresentam concentrações de $\mathrm{Pb}$ e de outros elementos, maiores que as da superfície do corpo líquido (Vaz, 1996).
- Não há correlação com as variáveis: alcalinidade, $\mathrm{DQO}$ e $\mathrm{C}_{\mathrm{Pb}(\mathrm{a})}$.

Análise da influência do local de amostragem: A Tabela 05 apresenta as concentrações médias de $\mathrm{Pb}$ total na água $\mathrm{C}_{\mathrm{Pb}(\mathrm{ag})}$, ao longo dos 12 meses, para cada um dos 9 locais (pontos) de coleta de amostras, as temperaturas médias, os valores de $\mathrm{pH}$, e DQO, bem como, os valores das concentrações médias de $\mathrm{Pb}$ nos sedimentos $-\mathrm{C}_{\mathrm{Pb}(\mathrm{sd})}$ ao longo dos 12 meses, para cada um dos 6 locais de amostragem dos sedimentos. Aqui alerta-se ao leitor que os valores de concentração são diferenciados para a temporalidade e para o local de amostragem $\mathrm{C}_{\mathrm{Pb}(\mathrm{a})}, \mathrm{C}_{\mathrm{Pb}(\mathrm{s})}$ e $\mathrm{C}_{\mathrm{Pb}(\mathrm{ag})}, \mathrm{C}_{\mathrm{Pb}(\mathrm{sd})}$ respectivamente.

A Tabela 06 (1- correlação das variáveis do meio com $\mathrm{C}_{\mathrm{Pb}(\mathrm{ag})}$ ) apresenta as correlações negativas com o pH e com a temperatura. $\mathrm{E}$, não apresenta correlação com a alcalinidade e DQO, conforme análise também feita para a temporalidade. 
Tabela 07. Concentração total de alguns metais pesados e nutrientes no corpo de água do lago do Parque do Ingá, (Vaz, 1996).

\begin{tabular}{lccccccc}
\hline Local & $\begin{array}{c}\mathrm{Pb} \\
\left(\mu . \mathrm{L}^{-1}\right)\end{array}$ & $\begin{array}{c}\mathrm{Cu} \\
\left(\mu . \mathrm{L}^{-1}\right)\end{array}$ & $\begin{array}{c}\mathrm{Fe} \\
\left(\mathrm{mg} \cdot \mathrm{L}^{-1}\right)\end{array}$ & $\begin{array}{c}\mathrm{Mn} \\
\left(\mu . \mathrm{L}^{-1}\right)\end{array}$ & $\begin{array}{c}\mathrm{N} \\
\left(\mathrm{mg} . \mathrm{L}^{-1}\right)\end{array}$ & $\begin{array}{c}\mathrm{Ca} \\
\left(\mathrm{mg} . \mathrm{L}^{-1}\right)\end{array}$ & $\begin{array}{c}\mathrm{K} \\
\left(\mathrm{mg} . \mathrm{L}^{-1}\right)\end{array}$ \\
\hline $1^{*}$ & 20,88 & 0,32 & 0,64 & 17,17 & 1,20 & 5,01 & 1,55 \\
$2^{*}$ & 20,30 & 0,92 & 0,46 & 13,28 & 1,15 & 4,91 & 1,62 \\
$3^{*}$ & 16,82 & 0,40 & 0,40 & 13,28 & 1,05 & 4,99 & 1,63 \\
$4^{* *}$ & 22,87 & 3,30 & 1,36 & 71,86 & 1,27 & 5,42 & 1,81 \\
$5^{*}$ & 13,45 & 0,00 & 0,43 & 13,45 & 0,95 & 4,64 & 1,58 \\
$6^{* *}$ & 29,05 & 13,25 & 1,38 & 53,60 & 1,43 & 5,58 & 1,69 \\
$7^{*}$ & 17,15 & 0,33 & 0,40 & 13,56 & 1,18 & 4,73 & 1,68 \\
$8^{* *}$ & 20,18 & 6,90 & 2,77 & 128,54 & 2,80 & 6,28 & 2,08 \\
$9^{*}$ & 12,35 & 0,68 & 0,45 & 12,64 & 1,08 & 4,66 & 1,79 \\
\hline
\end{tabular}

* - amostras de superfície; ** - amostras de fundo (50 cm do fundo do lago).

A correlação das variáveis com $\mathrm{C}_{\mathrm{Pb}(\mathrm{sd})}$ conforme parte 2 da Tabela 06, com exceção do $\mathrm{pH}$, repete-se a mesma dependência que no estudo da temporalidade. Pela mesma Tabela observase haver uma correlação fortemente positiva da concentração de $\mathrm{Pb}$ nos sedimentos - $\mathrm{C}_{\mathrm{Pb}(\mathrm{sd})} \mathrm{com}$ a concentração de $\mathrm{Pb}$ na água $-\mathrm{C}_{\mathrm{Pb}(\mathrm{ag})}$.

Outros fatores de análise: $O$ corpo d'água encontra-se estratificado nos seus locais mais profundos, fato verificado pela medida da temperatura do corpo de água (Vaz, 1996) e confirmado pelas concentrações de alguns metais pesados e nutrientes: $\mathrm{Pb}, \mathrm{Cu}, \mathrm{Fe}, \mathrm{Mn}, \mathrm{N}$, $\mathrm{Ca}$ e $\mathrm{Mg}$, conforme Tabela 07 e medidas da alcalinidade e DQO, conforme Tabela 04 (Vaz, 1996), onde se verifica que os valores das respectivas concentrações nas amostras de superfície são menores que as das amostras de profundidade.

Considerando os limites preconizados pelo CONAMA de 30 e 50 ng.mL ${ }^{-1}$ para águas destinadas à irrigação, sedentação de animais e aquicultura, verifica-se pelos valores médios mensais da concentração de $\mathrm{Pb}$ na água (Tabela 03), que o lago não apresenta problemas.

Pelos valores das concentrações de $\mathrm{Pb}$ na água, Tabela 05, nos locais 1 e 2 (entrada) e 9 (saída do lago) observa-se que há uma fração que fica no lago. Fato confirmado também pela diminuição da concentração de $\mathrm{Pb}$ nas amostras de superfície (locais 3, 5 e 7) e aumento da concentração de $\mathrm{Pb}$ nas amostras de fundo dos respectivos pontos 4,6 e 8 .

\section{CONCLUSÃO}

Pelo estudo realizado conclui-se:

- Em nível de 5\% e 1\% de significância: a) a concentração de chumbo total no corpo d'água não depende do local da coleta. Contudo, depende do período que foi realizada a mesma; b) a concentração de chumbo nos sedimentos, depende do local e do período da coleta.

- Com relação ao período de coleta, em nível de 5\% de significância, verificou-se que:

a) o índice pluviométrico (I.P.) tem correlação positiva com a concentração de chumbo na água e negativa com a concentração de $\mathrm{Pb}$ nos sedimentos; b) na água não se verificou nenhuma correlação entre as variáveis $\mathrm{pH}$, temperatura, alcalinidade e DQO com a concentração de chumbo; c) nos sedimentos verificou-se uma correlação positiva entre o $\mathrm{pH}$ e a temperatura do corpo d'água com a concentração de $\mathrm{Pb}$ nos mesmos. $\mathrm{E}$, as variáveis: alcalinidade, $\mathrm{DQO}$ e $\mathrm{C}_{\mathrm{Pb}(\mathrm{a})}$ não apresentaram nenhuma correlação. 
- Com relação ao local de coletas, com o mesmo nível de significância, verificou-se que:

a) na água e nos sedimentos o $\mathrm{pH}$ e a temperatura apresentaram correlações negativas e a alcalinidade e DQO não se correlacionaram com a concentração de $\mathrm{Pb}$, na água e no sedimento; b) a concentração de $\mathrm{Pb}$ no sedimento tem correlação positiva com a concentração do $\mathrm{Pb}$ na água, o que não aconteceu com estas variáveis no período das coletas;

- As concentrações de chumbo na água estão abaixo dos valores limites recomendados pelo CONAMA.

- O corpo d'água do lago, em suas partes mais profundas está estratificado.

\section{AGRADECIMENTOS}

A equipe de trabalho agradece ao Departamento de Química, ao Grupo de Estudos Multidisciplinares do Ambiente - GEMA, ao Núcleo de Pesquisas em Limnologia, Ictiologia e Aquicultura - NUPELIA da Universidade Estadual de Maringá, às Instituições UFPR, PUC-PR e CEFET-PR de Curitiba e à CAPES pelo apoio recebido.

\section{RESUMO:}

Este trabalho analisa a dinâmica do chumbo no lago do parque do Ingá, Maringá - PR. Mensalmente durante um ano foram coletadas amostras de água e sedimentos e o pH e temperatura medidos no ato da coleta. Nas amostras de água foi determinada a Demanda Química de Oxigênio (DQO) e a concentração total de $\mathrm{Pb}$ na água e sedimentos. A determinação da concentração $\mathrm{Pb}$ na água $\mathrm{e}$ sedimentos foi feita com a técnica da espectrometria da absorção atômica, modalidade chama. Os valores médios mensais encontrados, em intervalos e médias globais foram respectivamente: concentração de $\mathrm{Pb}$ na água, em ng.mL ${ }^{-1}$, não detectado (nd) - 55,56 e 19,23; $\mathrm{Pb}$ em sedimentos, em $\mu \mathrm{g} \cdot \mathrm{g}^{-1}, 48,73-92,87$ e 71,$93 ; \mathrm{pH}, 7,02-8,23$ e 7,60; temperatura, em
${ }^{0} \mathrm{C}, 19,33$ - 27,48 e 23,73; DQO, em mg. $\mathrm{L}^{-1}$, 13,33 - 30,11 e 23,54. Uma análise dos resultados das variáveis medidas, em nível de $5 \%$ de significância permitem concluir que a concentração de $\mathrm{Pb}$ na água é independente dos pontos de coleta (local), mas, dependente do período de amostragem (temporalidade) e nos sedimentos dependente dos dois parâmetros.

\section{REFERÊNCIAS BIBLIOGRÁFICAS}

Adriano, D.C. (1986), Trace Elements in the Terrestrial Environment. Berlin: SpringerVerlag, p. 219 - 262.

Agemian, H. \& Chau, A.S.Y. (1975), An atomic absorption method for the determination of 20 elements in lakes sediments after acid digestion. Anal. Chim. Acta, 80, 61 - 66, 1975.

Analytical Methods committee (1987), Recommendations for definition, estimation and use of the detection limit. Analyst, 112, 199 - 204, 1987.

Bartram, J. \& Ballance, R. [Editors] (1996), Water Quality Monitoring. London: E \& FN SPON (Chapman \& Hall), 383 p.

Boekx, R.L. (1986), Lead Poisoning in Children. Anal. Chem., 58(2), 274A - 287A, 1986.

Braile, P.M. \& Cavalcanti, J.B.W.A. (1979), Manual de Tratamento de Águas Residuárias e Industriais. São Paulo: Companhia de Tecnologia e Saneamento Ambiental (CETESB), p. 42 - 44.

Chow, T.J. \& Patterson, C.C. (1962), The occurence and significance of lead isotopes in pelagic sediments. Geochim. Cosmochim. Acta, 26, 263 - 308, 1962.

Costa Neto, P.L.D. (1977), Estatística. São Paulo: Editora Edgard Blücher Ltda, p. 152 177.

Craig, P.J. (1980), Metal cycles and Biological Methylation. In: Hutzinger, D. - The Handbook of Environmental and Biogeochemical Cycles. Berlin: SpringerVerlag, p. 170 - 227.

Daines, R.H; Motto, H. \& Chilko, D.M. (1970), Atmospheric lead: Its relationship to traffic volume and proximity to highways. Environ. Sci. \& Technol., 4(4), 318-322, 1970. 
Davies, B.E. (1990), Lead. In: Alloway, B. J. Heavy metals in soils. London: Blackie and John Wiley \& Sons, Inc; p. 177 - 194.

Durum, W.H. \& Haffty, J. (1963), Implications of the minor element content of some major streams of the world. Geochim. Cosmochim. Acta, 27, 01 - 11, 1963.

Gomes, F.P. (1987), Curso de Estatística Experimental. 12. ed., São Paulo: Livraria Nobel S. A., 467 p.

Horne, R.A. (1969), Marine Chemistry. New York: John Wiley \& Sons, Inc., p. 361 - 435.

Horwitz, W. [Editor] (1980), Official Methods of Analysis of the Association of Official Analytical Chemists (A.O.A.C). 13. ed. Washington: Published by the A.O.A.C., p. $557-559$.

Kabata-Pendias, A. \& Pendias, H. (1992), Trace Elements in Soils and Plants. 2. ed. London: CRC Press, Inc., p. 71 - 76.

Karin, R.W.; Buono, J.A. \& Faching, J.L. (1975), Removal of trace elemental impurities from polyethylene by nitric acid. Anal. Chem. 47 (13), 2296 - 2299, 1975.

Keith, L.H. [Editor] (1996a), Principles of Environmental Sampling. 2. ed. Washington: Americam Chemical Society, 648 p.

Keith, L.H. [Editor] (1996b), Compilation of EPA's Sampling and Analysis Methods. 2. ed. New York: Lewis Publishers, 1691 p.

Kirk-Othmer Encyclopedia of Chemical Technology (1981), 3. ed. New York: John Wiley Sons, v. 14, p. 98 - 200.

Klaassen, C.D. (1991), Metais pesados e seus antagonistas. In: Gilman, A.G.; Goodman, L.S. E Gilman, A. - As Bases Farmacológicas da Terapêutica, 8. ed. Rio de Janeiro: Editora Guanabara Koogan S.A., p. $1061-1065$.

Krishnamurty, K.V.; Shpirt, E. \& Reddy, M.M. (1976), Trace metal extraction of soils and sediments by nitric acid - hydrogen peroxide. At. Absorpt. Newsl., 15(3), 68 - 70, 1976.

Lee, J.D. (1980), Química Inorgânica. São Paulo: Editora Edgard Blücher Ltda., p. 484.

Lewis, R.J. (1996), Sax's Dangerous Properties of Industrial Materials, 9. ed. New York: Van Nostrand Reinhold, v. III, p. 2032, 1996.

Mart, L. (1979), Prevention of Contamination and other accuracy risks in voltammetric trace metal analysis of natural waters. Part I.
Preparatory Steps, filtration and storage of waters samples. Fresenius Z. Anal. Chem. 296, 350 - 357, 1979.

McGraw-HilL Encyclopedia of Environmental Science (1980), 2. ed. New York: McGRAW-HILL Book Company, p. 349 357.

Moody, J.R. \& Lindstrom, R.M. (1977), Selection and cleaning of plastic containers for storage of trace element samples. Anal. Chem. 49(14), 2264 - 2267, 1977.

Moore, J.W. \& Ramamoorthy, S. (1984), Heavy Metals in Natural Waters. Berlin: SpringerVerlag, p. 100 - 124.

Patterson, C.C. \& Settle, D.M. (1976), The reduction of orders of magnitude errors in lead, analysis of biological materials and natural waters by evaluating and controlling the extent and sources of industrial lead contamination introduced during sample collecting and analysis. In: NBS SPECIAL PUBLICATION 422 - Accuracy in trace analysis. Sampling, sample handling, and analysis. Washington. US Government Printing Office, p. 321 - 351.

Polprasert, C. (1982), Heavy metal pollution in the Chao Phraya river estuary, Thailand. Water Research. 16, 775 - 784, 1982.

Purves, D. (1985), Trace - Element Contamination of the Environment. Rev. ed. Amsterdam: Elsevier Science Publishing Company Inc., p. 57 - 61.

Rodricks, J.V. (1995), Calculated Risks. Cambridge-Great Britain: Athenaeum Press Ltd., p. 82 and 99-100.

Sax, N.I. (1979), Dangerous Properties of Industrial Materials. 5. ed. New York: Van Nostrand Reinhold Company, p. 765 - 771.

Schuck, E.A. \& Locke, J.K. (1970), Relationship of automotive lead particulates to certain consumer crops. Environ. Sci. Technol., 4(4), 325 - 330, 1970.

Sinex, S.A.; Contillo, A.Y. \& Helz, G.R. (1980), Accuracy of extraction methods for trace metals in sediments. Anal. Chem., 52, 2342 2346, 1980.

Smith, R.G.; Szajnar, J. \& Hecker, L. (1970), Study of lead levels in experimental animals. Environ. Sci. Technol., 4(4), 333 - 338, 1970.

Souza, H.B. \& Derísio, J.C. (1977), Guia Técnico de Coleta de Amostras de Águas. 
São Paulo: Companhia de Tecnologia de Saneamento Ambiental - CETESB, 245 p.

Taylor, S.R. (1965), Abundance of chemical elements in the continental crust: a new table. Geochim. Cosmochim. Acta, 28, 1273 1285.,1965.

Vaz, S.R (1996), Estudo de aspectos químicos e físico-químicos do lago do Parque do Ingá. Dissertação de Mestrado. Departamento de Química da Universidade Estadual de Maringá, Maringá, Paraná, Brasil.

Wagner, R. (1976), Sampling and sample preparation - water. Fresenius Z. Anal. Chem., 282, 315 - 321, 1976.
Welz, B. (1985), Atomic Absorption Spectrometry. 2. ed. Weinheim-Germany: VCH Verlagsgesellschaft, p. 107 - 127.

WMO - World Meteorological Organization (1988), Manual on Water-Quality Monitoring. Operational Hydrology Report $\mathrm{N}^{0}$ 27. Geneva: Secretariat of the World Meteorological Organization, 197 p.

Received: September 19, 1997; Revised: October 19, 1997; Accepted: March 12, 1998. 